\title{
Towards Automated Specifications of Scenarios in Enhanced Learning Technology
}

\author{
A. Rius Gavidia, UOC Open University of Catalonia, Spain \\ M. A. Sicilia Urbán, UAH University of Alcalá, Spain \\ E. García-Barriocanal, UAH University of Alcalá, Spain \\ G. Macarro Palazuelos, UAH University of Alcalá, Spain
}

\begin{abstract}
Recent standardization efforts in e-learning technology have resulted in a number of specifications, however, the automation process that is considered essential in a learning management system (LMS) is a less explored one. As learning technology becomes more widespread and more heterogeneous, there is a growing need to specify processes that cross the boundaries of a single LMS or learning resource repository. This article proposes to obtain a specification orientated to automation that takes on board the heterogeneity of systems and formats and provides a language for specifying complex and generic interactions. Having this goal in mind, a technique based on three steps is suggested. The semantic conformance profiles, the business process management (BPM) diagram, and its translation into the business process execution language (BPEL) seem to be suitable for achieving it.
\end{abstract}

Keywords: $\quad$ automated specification processes; learning technology scenarios; learning management systems

\section{INTRODUCTION}

The IMS digital repositories interoperability (DRI) specification (IMS Global Consortium, 2003) suggests recommendations for the interoperation of the most common repository functions. This specification acknowledges that a wide range of content formats, implemented systems, technologies, and established practices already exist; it is tied to IMS specifications on the contents, but it does not assume the hetero- geneity of the systems and formats, and does not provide a language for specifying complex, generic interactions.

In order to achieve interoperability among systems and formats, the eduSource Canada project has designed and implemented a standard communication protocol, ECL (Eap, Hatala, \& Richards, 2004). The ECL protocol is flexible with respect to metadata schemas and repository contents, and it allows new 
and existing repositories to communicate and share resources across a network. It conforms to IMS DRI specifications and implements its main functions, and, furthermore, it extends the IMS protocol with some definitions based on the OAI harvesting protocol. Also conforming to IMS DRI specification, the PAWSEL project proposes an architecture that intends to facilitate the heterogeneous conversational patterns among participants of the scenario (Macarro et al., 2006). The intermediary level provides the orchestration skills for the rest of the components through service interfaces and the relation among learning services. Their users and intermediaries are understood as scenarios. The SleD2 project presents another architecture that facilitates the integration of learning services instead of Web services. The CooperCore engines such as the IMS LD service are integrated into a workflow engine and satisfy the automation of scenarios but are limited to the delivery of activities (Vogten, Martens, Nadolski, Tattersall, Van Rosmalen, \& Koper, 2006)

The term scenario in the e-learning area, and the reutilization of learning objects (LOs) as a scenario-based approach were introduced in an attempt to present the learning-objects metadata as required infrastructure to support some LMS (learning management system) functions (Sicilia \& Lytras, 2005). Also, in relation to scenarios, it has been proposed to use the semantic conformance profile (SCP) toward the automation processes (Sicilia, Pagés, García, Sánchez-Alonso, \& Rius, 2004). Taking into account this previous work (Macarro et al., 2006; Sicilia \& Lytras; Sicilia et al.), and acknowledging the need for having a language for processes specification to describe the fundamental processes in the LMS and all the interactions among the participants, we propose BPMN (business process modeling notation) as the most suitable one. Subsequently, we state the main advantages that BPMN brings about to e-learning.

- It supports the exchange formats between applications: The serialization of
BPMN is done for XML(extensible markup language) exchange. A comparative study (Mendeling, Neumann, \& Nüttgens, 2004) of about 15 different XML-based specifications for BPM (business process management) concludes that BPMN and BPEL (business process execution language) are the languages that satisfy the majority of the items that assure the exchange formats and the interoperability.

- It reduces heterogeneity among LMS: BPMN is accepted by the business community to describe the processes' workflows. It can also be used in an e-learning context as a specification language in order to reduce the heterogeneity among the specification techniques and bring interoperability between different systems (Object Management Group [OMG], 2006). The justification can be found in Helic, Hrastmik, and Maurer (2005).

- It provides elements to specify complex and generic interactions: To achieve dynamic and adaptive LMSs, their processes have to be specified in a language that will be able to (a) describe in a standard graphical and understandable notation, (b) define abstract modeling elements by means of a metamodeling notation, and (c) offer different techniques of modeling processes for one participant or more that are connected through the flow messages (OMG, 2006).

- It provides support to the generation of executable specifications: BPMN has an internal model that enables the generation of BPEL executable specifications with automated support. It contributes to reduce the number of errors introduced during the translation phase, decreasing costs of the development and increasing productivity. In a general sense, it can be considered in a normative way as a bridge for the gap between business process design and process implementation (OMG, 2006; Stephen, 2004). 
9 more pages are available in the full version of this document, which may be purchased using the "Add to Cart" button on the product's webpage:

www.igi-global.com/article/towards-automated-specificationsscenarios-enhanced/3001

\section{Related Content}

Game Informed Virtual Patients: Catalysts for Online Learning Communities and Professional Development of Medical Teachers

Michael Begg, David Dewhurst and Michael Ross (2010). Online Learning Communities and Teacher Professional Development: Methods for Improved Education Delivery (pp. 190-208).

www.igi-global.com/chapter/game-informed-virtual-patients/36941

Virtual School Science

Kevin F. Downing and Jennifer K. Holtz (2008). Online Science Learning: Best Practices and Technologies (pp. 30-48).

www.igi-global.com/chapter/virtual-school-science/27763

Using Lotus Learning Space to Enhance Student Learning of Data Communication

Michael W. Dixon, Johan M. Karlsson and Tanya J. McGill (2002). Web-Based Instructional Learning (pp. 163-171).

www.igi-global.com/chapter/using-lotus-learning-space-enhance/31346

What Factors Promote Sustained Online Discussions and Collaborative Learning in a Web-Based Course?

Xinchun Wang (2007). International Journal of Web-Based Learning and Teaching

Technologies (pp. 17-38).

www.igi-global.com/article/factors-promote-sustained-online-discussions/2977 